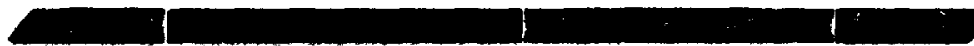

\title{
LUDWIG BOLTZMANN
}

\section{IA SUA OPERA E IL SUO PENSIERO}

\section{(20 febbraio 1844 = 7 settembre 1906).}

§ 1. Quando, sono appena tre anni, si celebrò con tanta solennità il sessantesimo anniversario del professor Ludwig Boltzmann, ricordo di essermi meravigliato in apprendere che egli fosse relativamente cosi giovine. In realtá il Boltzmann apparteneva per l'opera e per il pensiero a quella grande generazione, che vide nascere la termodinamica e la nuova elettrologia, alla generazione di Helmholtz e di Clausius, di Lord Kelvin e di Maxwell.

La filosofia naturale dell'epoca moderna ha oscillato sempre, a cominciare dalle contese classiche dei continuatori di Newton coi discepoli di Cartesio, e venendo fino alle polemiche sempre vive dei meccanici e degli energetici contemporanei, fra due forme antagonistiche di pensiero e di ricerca. Si tengono stretti gli uni al buon metodo vecchio del nostro Galileo, al metodo che affionta un problema alla volta, e lo risolve, in tutte le sue càratteristiche quantitative; e gli altri confidano invece in una forma particolare di intuito, la quale dovrebbe porgere il mezzo per la scoperta e per l'enunciazione dei grandi principî generalissimi.

Questo non vual dire naturalmente che i singoli si rendano sempre bene conto della loro personale posizione filosofica. Newton, che pure aveva costruito un modello ingegnoso per l'interferenza della luce, enuncio una volta di sfuggita quell'hypotheses non fingo, che fu il mal seme della moderna 
fenomenologia; e l'ostwald ultimamente preconizzava sul serio «die Ueberwindung des wissenschaftlichen Materialismus », senza accorgersi che, nella ipotesi migliore, il resultato della sua battaglia sarebbe pur sempre quello di sostituire una dottrina semifilosofica ad un'altra, immeritevole anch'essa del vocabolo della scienza.

Ma Ludwig Boltzmann fu uno spirito troppo sottile, e troppo severamente educato e consapevole della importanza fondamentale del metodo nella ricerca scientifica ${ }^{1}$ ), per non scrutare egli stesso le sue convinzioni e le sue tendenze. $\mathbf{E}$ in un memorabile discorso, alla riunione di Monaco della Società dei Medici e Naturalisti Tedeschi (1899), diceva senza ambagi « Io mi presento a voi come un reazionario, che malgrado $i$ « novatori serba intatta la sua fede alle vecchie idee classi« che ». E veramente egli era persuaso che le imagini della fisica tradizionale, come mezzo euristico e come sussidio didatticn, meritassero sempre di venire coltivate con amore.

Egli avrebbe sorriso senza dubbio, se gli si fosse fatto l'appunto di costruire dei modelli piuttosto che delle vere e proprie teorje, avendo compreso con Maxwell che alle ricerche teoriche non può segnarsi altro scopo da quello in fuori della descrizione delle imagini o delle analogie meceaniche ${ }^{3} \%$

Perchè, conviene rilevarlo subito, se il Boltzmann fu un classico, lo si direbbe per le tendenze scientifiche un classico anglicizzante.

Egli raccontò una volta, che avendo richiesto di consiglio il suo maestro, lo Stefan, su le quistioni della fisica teorica, che gli conveniva approfondire, questi gli rispose ponendogli fra le mani una grammatica inglese. $\mathrm{E}$ il discepolo accolse

1) "Die Hauptursache des rapiden Fortschrittes der Naturwissenschaft in der letzten "Zeit liegt unzweifelhaft in der Auffinduıg und Yervollkommnung einer besonders geei"gneten Forschungsmethode

2) Meglio ancora "Allmahlich fanden die neuen Ideen in allen Gebieten Eingang. " Aus dem fiehiete der Wärmetheorie erwähne jch nur Helmholtz' berülımte Abhatidun-

"gen über die mechanischen Analogien des zweiten Hauptsatzes der Wärmetheorie. Ja,

" es zeirte sich, diss sie dela tojste der Wissensighaf besser entsprachen, als die alten

"Hypothesen und anch für den Forscher sellost bequemer waren. Denn dio alten Hy-

" pothesen konnten nur aufrecht erhaiten werden, so lange alles klappte; jetzt aber

" schadeten tinzelne Nichtübeseinstimmungen nicht mehr, denn einer blossen Analo-

"gie kann man es nicht ibel nehmen, wenn ste in einzelnen Punkten hinkt . 
quel monito e lo segui poi sempre nella sua rapida carriera luminesa. E all' influenza dello spirito e della coltura britannica dovette forse il Boltzmann l' equilibrio, che gli è riuscito di serbare in quarant'anni di lavoro fecondo, fra la speculazione teoretica e la sana attività sperimentale.

Una sua pagina su la natura e gli scopi della fisica teorica giova rileggere e meditare in Italia, mentre I'influenza di molteplici cause va rendendo sempre più crudo e doloroso il distacco fra i matematici e gli sperimentatori, e il Parlamento si prepara a consacrare in una legge quella che chiamano elegantemente la specializzazione della coltura.

«Die Physik ist heutzutage durch ibre vielen praktischen « Anwendungen populär geworden. Von der Tätigkeit eines « Mannes, der durch Versuche ein neues Gesetz in der Wir«kungsweise der Naturkräfte entdeckt oder auch bekannte «Gesetze bestätigt und erweitert, dürfte man sich eine Vor« stellung machen können. Aber was ist ein theoretischer «Physiker? Da letzterer gründliche mathematische Kenntnisse « besitzen muss, pflegt man seine Tätigkeit häufig die mathe«matische Physik zu nennen, jedoch nicht ganz entsprechend; "denn auch die Auswertung komplizierter physikalischer « Experimente, ja selbst die Lösung technischer Probleme, « kann weitschweifige und schwierige Rechnungen erfordern, " ist aber doch nicht der theoretischen Physik zuzuzählen. «Die theoretische Physik hat vielmehr, wie man früher sagte, « die Grundursachen der Erscheinungen aufzusuchen oder wie « man heute lieber sagt, sie hat die gewonnenen experimen«tellen Resultate unter einheitlichen Gesichtspunkten zusam« menzufassen, übersichtlich zu ordnen und möglichst klar « und einfach zu beschreiben, wodurch die Erfassung dersel« ben in ihrer ganzen Mannigfaltigkeit erleichtert, ia eigen« tlich erst ermöglicht wird. Deshalb wird sie in England « auch natural philosophy genannt».

Molti teorici pur troppo non mirano tanto alto, ma sembrano avere su la loro scienza l'opinione che Mefistofele professava a proposito della teologia 
"Am besten ist's auch hier, wenn ibr nur Einen hört,

* und auf des Meisters Worte schwört.

«Im Ganzen - haltet euch an Worte!

« dann geht ihr durch die sichre Pforte

«zum Tempel der Gewissheit ein».

Essi tengono in realtà un unico maestro, o, per essere più esatti, si restringono ad un solo indirizzo e continuano ad integrare da anni la medesima equazione differenziale. Eppure e bastato in questi ultimi tempi, che un matematico rivolgesse la sua attenzione ad un problema concreto, perchè subito ne risultassero illuminati molti fenomeni conosciuti, o se ne prevedessero di nuovi. É vero bensi, che quel matematico si chiamava Vito Volterra.

\$2. L'attività scientifica di Ludwig Boltzmann si è svolta quasi esclusivamente in tre campi : nella termodinamica prima e nella teoria cinetica dei gas, poi nella elettrologia maxwelliana, e da ultiıno nella meccanica propriamente detta. E ciò che resta di più originale e di più bello nell'opera del nostro Autore sono senza dubbio le indagini relative al secondo principio.

Carnot a suo tempo, e Clausius più tardi, avevano veduto in codesta proposizione una regola conforme ai resultati dell'esperienza, senza scrutarne peró le ragioni profonde, e senza saperla ridurre alle leggi più sicure della meccanica razionale; ma il genio di Helmholtz ha lasciato in questo campo una traccia luminosa. Le sue considerazioni ad ogni modo, fondate come erano su lo studio dei fenomeni ciclici, bastavano appena a descrivere quei processi, che in termodinamica banno nome di reversibili. E che le cose non potessero risultare diverse intende bene chi consideri che le equazioni di Lagrange sotto la seconda forma,

$$
\mathrm{Q}=\frac{d}{d t} \frac{\partial \mathrm{T}}{\partial \dot{q}}-\frac{\partial \mathrm{T}}{\partial q},
$$

non mutano affatto quando si faccia scorrere il tempo a ritroso. La irreversibilità resta al di fuori della meccanica classica. 
Helmholtz che fu grandissimo scienziato, e quindi conservatore di tendenze, non concluse di qui che si dovesse riformare la meccanica, ma piuttosto che i fenomeni irreversibili fossero tali rispetto a noi e rispetto ai mezzi di cui la fisica dispone. Era un'intuizione vaga che il Boltzmann precisò in una formola definitiva, affermando che il secondo principio della termodinamica è un teorema di calcolo delle probabilità.

Io non posso riportare in questo cenno tutte le classiche ricerche su la funzione $\mathrm{H}^{1}$ ), ma mi accontenteró di riassumerle in una forma sintetica, seguendo in parte un mirabile discorso «Ueber den zweiten Hauptsatz der mechanischen Wärmetheorie ${ }^{2}$ ).

La pressione esercitata da un gas su l'embolo che lo rinserra deriva da ció che, di volta in volta, l'una o l'altra molecola, con maggiore o minore vivacità, in direzione normale od obliqua, viene ad urtare la superficie dell' embolo. Ma il numero delle molecole che entrano in giuoco, è così grande che, sopra un elemento scelto a caso della superficie stessa, l' intensitá media degli urti rimane costante. La teoria cinetica preconizza dunque la meccanica statistica.

Se ora un sistema dato di corpi contiene una certa quantità di energia, questa non si tramuterá a caso di forma in forma, ma passerà anzi dalla forma meno probabile alla più probabile; che se la sua distribuzione fra i corpi del sistema non rispondeva da principio alle leggi delle probabilità vi si andrà poscia accostando per gradi.

Ma le forme di energia che si cerca di realizzare nella pratica sono appunto fra le meno probabili. Si vorrá, per esempio, spostare un corpo tutto insieme, o dare in altri termini alle molecole di cui risulta la medesima velocitá, che è il caso più improbabile fra tutti.

Una probabilitá maggiore corrisponde ad una forma più degralata di energia, ad una dunque sempre meno facilmente trasformabile.

1) Furono riccolte dal Boltzmann stesso nat primo volume delle sue "Vorlesungen übcr Gastheorie " (Leipzig, Barth, 1896).

2) L. Boltzniann. Populäre ichriften (Leipzig, Barth, 1905, pag. 25 e seg.). 
Se si prende un certo numero di palle bianche e un altro di palle nere e le si rimescolano con la mano, o si imaginano sottoposte comunque ad azioni variabili, si ritroveranno alla fine intimamente commiste. Un fatto analogo si verifica quando un corpo caldo viene a trovar'si in presenza di altri più freddi, $o$, che fa lo stesso, un sistema di molecole vivacemente agitate riesce in contatto intimo con altre molecole più lente. La distribuzione voluta dalle leggi delle probabilità deve stabilirsi a poco a poco e la temperatura riesce da ultimo uniforme.

Questa r'egola non esclude naturalmente la possibilità di utilizzare con artifizî opportuni la condizione improbabile, per ricavarne altre forme di energia, che non risulterebbero dal processo spontaneo. E così nella macchina a vapore si trasforma l'energia termica in meccanica, mentre una certa quantitá di calor'e degrada dall'alta alla bassa temperatura.

$\dot{E}$ riuscito al Boltzınann di assegnare per ogni sistema determinato una misura esatta (quantitativa) della probabilita corrispondente alle sue condizioni attuali, e, come si vede subito, codesto valore caratteristico (la funzione H) potrà servire di modello all'entropia classica del Clausius.

Si deve anzi affermare qualche cosa di più ogni funzione $f(\mathrm{H})$, per la quale la derivata $\frac{\partial f}{\partial H}$ conservi il suo segno nei limiti di variabilità necessarii, sará infatti utilizzabile al medesimo scopo.

II bel libro del Gibbs su la meccanica statistica costituisce in fondo lo svolgimento di codesto concetto; è dunque la descrizione di un certo numero di modelli, ugualmente accettabili (zulässig) dell'entropia termodinamica. Al quale proposito giova osservare come il Planck abbia stabilito, che la primitiva definizione del Boltzmann rimane in fondo la più semplice e la più opportuna (zweckmässig) fra le infinite definizioni possibili.

\$ 3. Se le ricerche teoriche più importanti del nostro Autore spettano al campo della termodinamica, le cose sue sperimentali di maggior conto ebbero invece di mira la soluzione 
di problemi relativi all' elettricità. E all' elettrologia maxwelliana in particolar'e.

A noi lontani sembra naturale questo interesse per una teoria di tanta importanza; ma quando il Boltzmann condusse i suoi lavori (1874), l'opinione del maggior numero era ben divel'sa da quella che ha voga al presente. Erano i tempi in cui i Fortschritte der Physik sbrigavano in 7 o 8 righe la recensione della grande memoria del Maxwell, accontentandosi di affermare che « ad ogni modo i resultati non ne erano diversi da quelli previsti dalle teorie ordinarie ».

Sotto l'influenza geniale dello Stefan, il Boltzmann seppe vedere invece che nell' opera del Maxwell qualche cosa di nuovo e di interessante ad ogni modo vi era, e si propose di verificare con una serie di misure dirette la relazione

$$
n=\sqrt{\bar{K}}
$$

Le sue ricerche portarono da prima su la costante dielettrica dei gas, e condussero ad un accordo estremamente buono, come si deduce dalle cifie che qui riferisco

\begin{tabular}{l|c|c}
\hline \multicolumn{1}{c|}{ Gas } & $n$ & $\sqrt{\mathrm{K}}$ \\
\hline & & \\
Aria & 1,000293 & 1,000295 \\
Idrogeno & 1,000139 & 1,000132 \\
Anidride carbonica & 1,000454 & 1,000473 \\
Ossido di carbonio & 1,000335 & 1,000345
\end{tabular}

È vero che Ayrton e Perry ricavarono dalle loro misure dei numeri, i quali non si accordano con la teoria altrettanto bene che quelli del Boltzmann, ma non è meno relo che il Klemenczicz, con un metodo completamente diverso, pitrovò molti anni più tardi (1885) i resultati del nostro Autore.

Quasi contemporaneamente attese il Boltzmann allo studio 
delle costanti dielettriche dello solfo, secondo le direzioni principali, e ne ottenne questi valori numerici

$\begin{array}{cccc} & 1 & 2 & 3 \\ n & 2,144 & 1,971 & 1,895 \\ V \overline{\mathrm{K}} & 2,185 & 1,992 & 1,952 ;\end{array}$

l'accordo non è più così buono, ma non poteva essere, come fu riconosciuto più tardi, per la natura stessa del problema. Nei corpi solidi infatti i raggi della luce si rifrangono alle volte secondo leggi quantitative diverse, da quelle che valgono per le onde più lunghe, sicchè in certi cristalli nemmeno le direzioni principali coincillono più.

Il Boltzmann tornò molti anni appresso all'elettrodinamica del Maxwell, con le sue « Vorlesungen über Maxwell's 'Theorie der Elektricität und des Lichtes $»^{1}$ ). Nelle quali cercó di svolgere dell'intera dottrina una esposizione rigorosa e sistematica. Il concetto dei moti ciclici, introdotto fin da principio, dà a queste lezioni una impareggiabile eleganza.

\$4. É una caratteristica sicura dei buoni spiriti quella di rifarsi di volta in volta alla considerazione dei postulati fondamentali della meccanica. Da Galileo e Newton fino a Helmholtz e Hertz nessuno è sfuggito alla regola generale, e nemmeno Ludwig Boltzmann forma eccezione. L'impulso gli venne dall'apparire postumo dei Principî del Hertz; pure professando l'ammirazione più profonda per le idee originali del grande pensatore, ritenne infatti il Boltzmann che la nuova esposizione della meccanica non andasse scevra di difficoltà. Hertz sopprimeva come è noto il concetto della folza, e lo sostituiva con quello dei moti e delle masse nascoste, ma la morte immatura non gli permise pur troppo di precisare le sue idee; e nessun matematico dopo di lui ha saputo portare avanticon profitto l'opera ponderosa.

Guidato dalle sue tendenze e dalle abitudini conservatrici dello spirito il Boltzmann vide invece un altro lato del pro-

1) Leipzig, Barth, 1891 e 1899. 
blema; egli pensó che non fosse venuto ancora il tempo di abbandonare le forme tradizionali, e che la meccanica classica, pure adattandosi ai nuovi e più rigidi postulati della teoria della conoscenza, potesse anzi soccorrere come per il passato ai fini dell'insegnamento e della ricerca.

Frutto di queste sue meditazioni furono i due volumi delle «Vorlesungen über die Prinzipe der Mechanik» ${ }^{1}$ ). Dei quali volumi, se pure non è cospicuo l'elemento nuovo ed originale, merita di essere citata a modello la forma, per il rigore e la sobrietà.

Muove il Boltzman da sette postulati, e costruisce su di essi l'intero edifizio della meccanica; e $\mathrm{i}$ postulati suoi sono di duplice natura. I primi due introducono l'idea degli atomi, i cinque rimanenti fissano il concetto dell'accelerazione e quello della massa.

Il fatto dell' avere egli stabilito fin da principio, e come fondamentale, l'ipotesi atomistica, merita di essere posto in evidenza, perchè rispondeva ad una convinzione del Boltzmann, maturamente radicata. Era persuaso infatti il nostro Autore che del concetto degli atomi non si potesse fare a meno in nessun modo nelle scienze naturali, e un articolo «Ueber die Unentbehrlichkeit der Atomistik in der Naturwissenschaft ${ }^{2}$ ) rimane fra le cose sue più interessanti e piư belle.

Dimostra il Boltzmann in questo lavoro che il processo fenomenologico, secondo il quale si dovrebbero assumere delle equazioni differenziali come ipotesi prime, riposa in fondo sopra un equivoco, perchè l'operazione stessa del derivare im. plica un passaggio al limite sopra una serie discreta di valori. Il concetto della discontinuità è dunque implicito nei procedimenti del calcolo, e male si saprebbe come metterlo da banda.

\$ 5. To mi sono limitato finora alle cose più fondamentali che del Boltzmann ci restano, ma le ricerche minori, su le quali mi è giuocoforza sorvolare, basterebbero da sole alla fama durevole di uno scienziato. Il suo spirito sempre desto

1) Leipzlg, Barth, 1897 e 1904 .

2) Wied. Ant. LX, 231, 1897. 
ed acuto si rivolse infatti a molti altri campi, lasciando in tutti una traccia profonda. Egli si è occupato della capillarità e della polarizzazione rotatoria, del diamagnetismo e dell' elettrostrizione, dell'elasticità susseguente e del fenomeno di Hall.

Professore di meccanica e di fisica matematica, di fisica sperimentale e di filosofia, fu grande scienziato senza essere specialista, o forse appunto perchè non era specialista.

ANTonio Garbasso. 